\title{
Two-dimensional photonic crystal accelerator structures
}

\author{
Benjamin M. Cowan* \\ Stanford Linear Accelerator Center, Stanford University, Stanford, California 94309, USA
}

(Received 18 July 2003; published 2 October 2003)

\begin{abstract}
Photonic crystals provide a method of confining a synchronous speed-of-light mode in an alldielectric structure, likely a necessary feature in any optical accelerator. We explore computationally a class of photonic crystal structures with translational symmetry in a direction transverse to the electron beam. We demonstrate synchronous waveguide modes and discuss relevant parameters of such modes. We then explore how accelerator parameters vary as the geometry of the structure is changed and consider trade-offs inherent in the design of an accelerator of this type.
\end{abstract}

DOI: 10.1103/PhysRevSTAB.6.101301

PACS numbers: 41.75.Jv, 42.70.Qs

\section{INTRODUCTION}

The extraordinary electric fields available from laser systems make laser-driven charged particle acceleration an exciting possibility. However, simply scaling down traditional microwave accelerator structures to optical frequencies would not be practical due to the low breakdown threshold of metals at such frequencies [1]. To achieve linear laser-driven acceleration in vacuum, therefore, a structure composed entirely of dielectric materials is required.

Designs of linear vacuum laser accelerator structures generally fall into one of two categories: macroscopic, far-field structures and near-field, guided-mode structures. Because they are relatively easy to fabricate and diagnose, macroscopic structures have generally been used in vacuum laser acceleration experiments, such as LEAP [2,3]. On the other hand, guided-mode structures have the advantage of a smaller mode volume and therefore more efficient coupling of laser radiation to a particle beam. Since both types of structures have gradients limited by the damage threshold of the optics, guided-mode structures may attain higher gradient. A scaling law applies to the ratio of on-axis accelerating field to electric field magnitude, given by $E_{z} /|\mathbf{E}| \sim \lambda / w_{0}$, where $\lambda$ is the wavelength and $w_{0}$ is the transverse laser mode size. With $|\mathbf{E}|$ limited by the damage threshold of the optics, a structure with a smaller mode size can sustain a larger accelerating gradient.

These considerations suggest using near-field, guidedmode structures. Photonic crystals [4] provide a means of guiding a speed-of-light optical mode in an all-dielectric structure, and a synchronous mode has been shown to exist in a photonic crystal fiber [5]. Photonic crystals have also been investigated as possible accelerator structures at microwave frequencies [6,7]. In fact, photonic crystals can support accelerating modes in a variety of structures, and we explore a particular class of geometries below.

*Electronic address: benc@slac.stanford.edu

\section{ACCELERATING MODES IN PHOTONIC CRYSTALS}

The geometries we consider here are two dimensional: we take them to be infinite in the vertical (y) direction, transverse to the electron beam, which we take to propagate in the $z$ direction (see Figs. 1 or 3 for a description of the coordinate system). As such, these structures would not be suitable for actual acceleration unless a method of vertical confinement were found, and then their properties would be altered from those computed here. Rather, they provide a means to build intuition for photonic crystal structures. By considering two-dimensional structures our computation time is greatly reduced, allowing us to

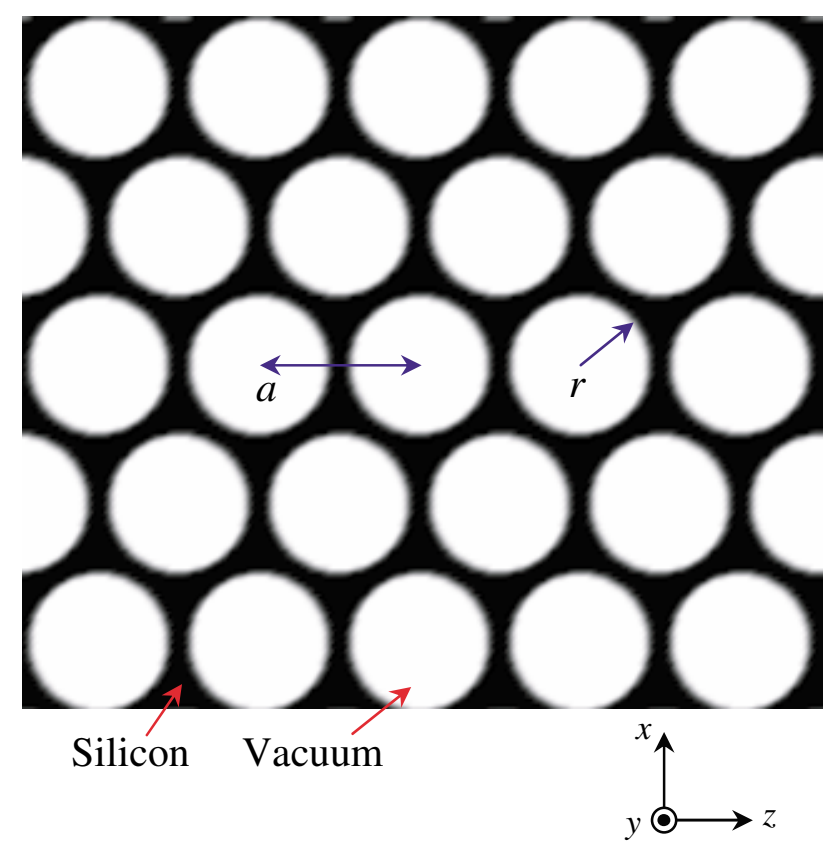

FIG. 1. (Color) The geometry of an optimized 2D photonic crystal lattice consisting of vacuum holes in silicon. Here $r=$ $0.427 a$. The coordinate system used throughout this paper is shown, and we take the particle beam to propagate in the $z$ direction. 
quickly explore multiple sets of geometric parameters. The computational technique is discussed at the end of this section, after presenting the structure geometry.

\section{A. Lattice geometry}

Our underlying photonic crystal lattice is a triangular array of vacuum holes in a silicon substrate, shown in Fig. 1.

We consider laser acceleration using a wavelength of $1.5 \mu \mathrm{m}$, in the telecom band where many promising sources exist. At this wavelength silicon has a normalized permittivity of $\epsilon_{r}=\epsilon / \epsilon_{0}=12.1$ [8]. As shown in the figure, the holes have radius $r$, and the lattice constant is $a$.

Because of the vertical symmetry of the structure we can classify each mode as either TE or TM, where the "transverse" designation is with respect to the $y$ direction, not the $z$ direction in which the beam propagates. Thus the accelerating modes are TE since these have the $\mathbf{E}$ field in the plane of the structure.

Like electronic states in a solid, electromagnetic modes in the lattice fall in discrete bands. The TE band structure of the lattice is shown in Fig. 2.

As described in [4], the irreducible Brillouin zone of this lattice forms a triangle in reciprocal space. This triangle has corners at $\mathbf{k}=0$ (the $\Gamma$ point), $\mathbf{k}=$ $(2 \pi / a)(\hat{\mathbf{x}} / 2)$ (the $M$ point), and $\mathbf{k}=(2 \pi / a)(\hat{\mathbf{x}} / 2+$ $\hat{\mathbf{z}} / 2 \sqrt{3}$ ) (the $K$ point). The figure shows the frequencies for values of $\mathbf{k}$ around the edge of the irreducible Brillouin zone, for $\Gamma$ to $M$ to $K$ and back to $\Gamma$. We see from this figure that the lattice exhibits a band gap-a range of frequencies in which no TE mode exists. In fact, this lattice was chosen specifically to exhibit such a gap based on energy considerations; also, a high-index material was chosen in order to obtain a wide band gap [4].

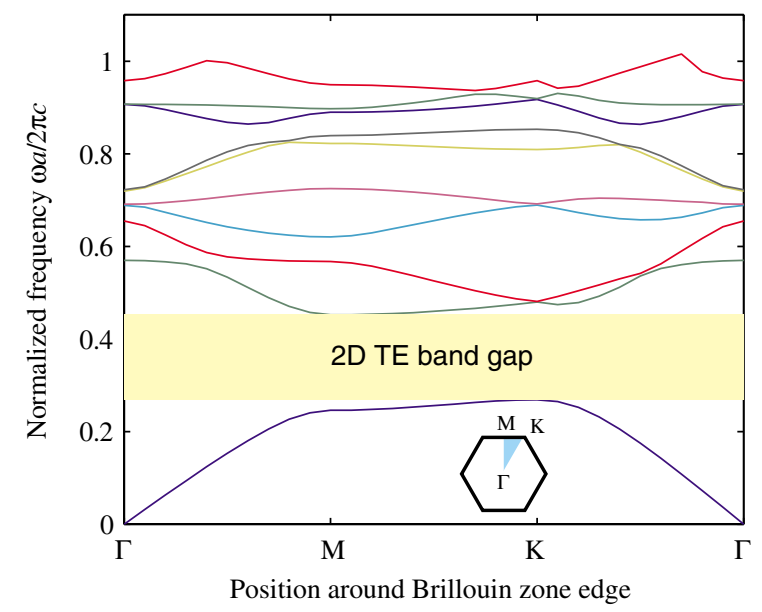

FIG. 2. (Color) The TE band structure of the photonic crystal lattice shown in Fig. 1, with $r=0.427 a$. The symbols $\Gamma, M$, and $K$ refer to the points at the corners of the irreducible Brillouin zone, as shown in the inset.
Then, $r / a$ was optimized to give the widest band gap, which occurs at $r / a=0.427$. With light at frequencies in the band gap forbidden to propagate in the lattice, modes at such frequencies can exist only in a defect, should one be provided. Thus we are able to create waveguides in alldielectric photonic crystal structures and customize them to support accelerating modes. In addition, such a waveguide only confines light at frequencies in the band gap, so higher-order guided wakefield modes are suppressed, potentially eliminating a major source of beam breakup instability.

\section{B. Accelerator structure geometry}

Our example accelerator structure consists of the lattice shown in Fig. 1 with a vacuum waveguide of width $w$ introduced. The waveguide geometry is shown in Fig. 3.

Specifically, $w$ is defined so that the distances between the centers of the holes adjacent to the waveguide are $w+a$. In addition, we can line the edge of the waveguide with dielectric, a concept similar to the dielectric waveguide accelerator described in [9]. We define $\delta$ as the total width of silicon in the guide, so there is $\delta / 2$ of dielectric "padding" on each edge.

If we normalize the length scales of our structures to the lattice constant $a$, we are left with three parameters: the guide width $w$, the pad width $\delta$, and the wavelength. Fixing the lattice constant makes the wavelength, or equivalently the longitudinal wave number $k_{z}$ or frequency $\omega$, a free parameter. For a general selection of $w$ and $\delta$, there will be a $k_{z}$ for which the waveguide mode is synchronous, i.e., $\omega=c k_{z}$. (Certainly if there is too much dielectric material within the waveguide, the phase velocity will be limited below $c$; consider for instance the

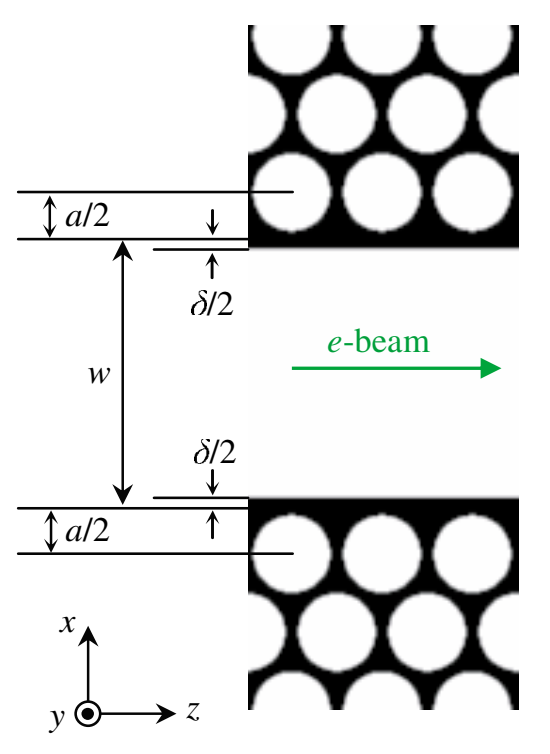

FIG. 3. (Color) The waveguide design, illustrating the geometric parameters. Here $r=0.427 a, w=3.0 a$, and $\delta=0.25 a$. 
(a) Varying $w, \delta=0$

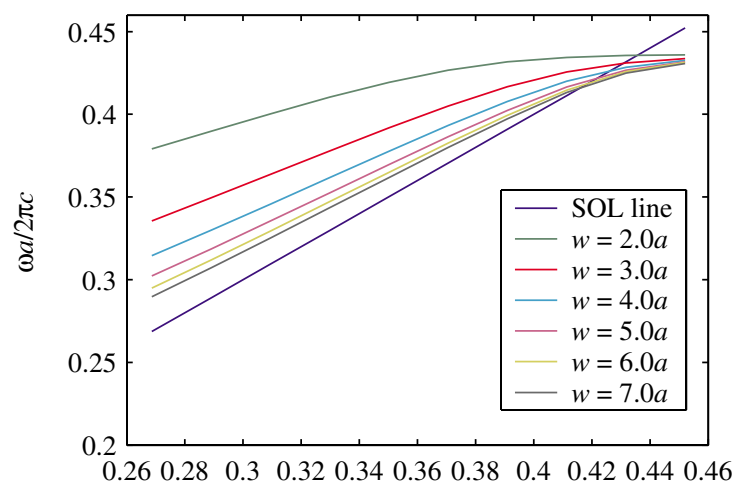

(b) Varying $\delta, w=3.0 a$

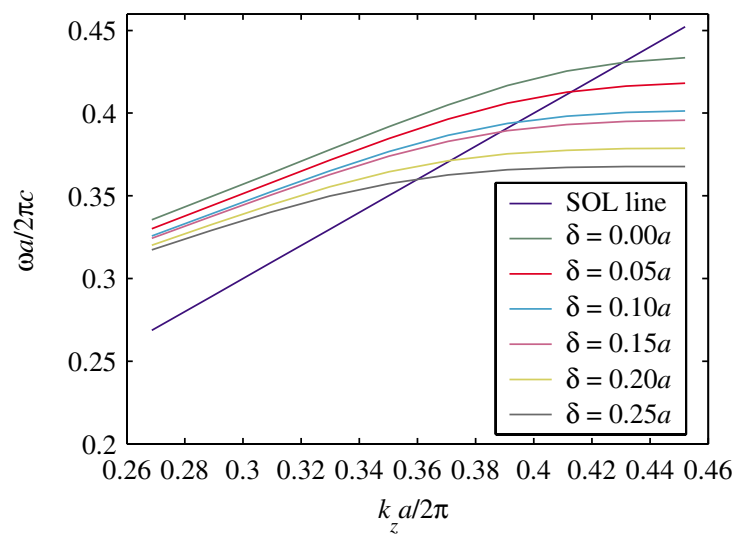

FIG. 4. (Color) Dispersion curves for a set of waveguide geometries, plotted with the speed-of-light (SOL) line shown. The range of wave numbers consists of all $k_{z}$ with $c k_{z}$ a frequency in the band gap. (a) We fix $\delta=0$ and vary $w$; (b) we fix $w=3.0 a$ and vary $\delta$.

case of a guide entirely filled with silicon.) This is demonstrated in Fig. 4(a), which shows the dispersion diagrams for a set of waveguide widths, with $\delta=0$.

Since each curve crosses the speed-of-light line, each geometry supports a synchronous mode. Adding dielectric material to the edges of the guide brings the speedof-light frequency down into the interior of the band gap, as shown in Fig. 4(b). We show such a mode with its longitudinal electric field in Fig. 5, for $w=3.0 a$ and $\delta=0.25 a$.

Observe that the mode is mostly confined to within $a$ of the edge of the guide; the wide band gap makes such good confinement possible.

\section{Computation techniques}

For the photonic crystal computations we use the MIT Photonic-Bands (MPB) package, a public-domain code using an iterative eigensolver technique [10]. For a given geometry and Bloch wave vector, MPB computes frequencies and field configurations of supported modes.

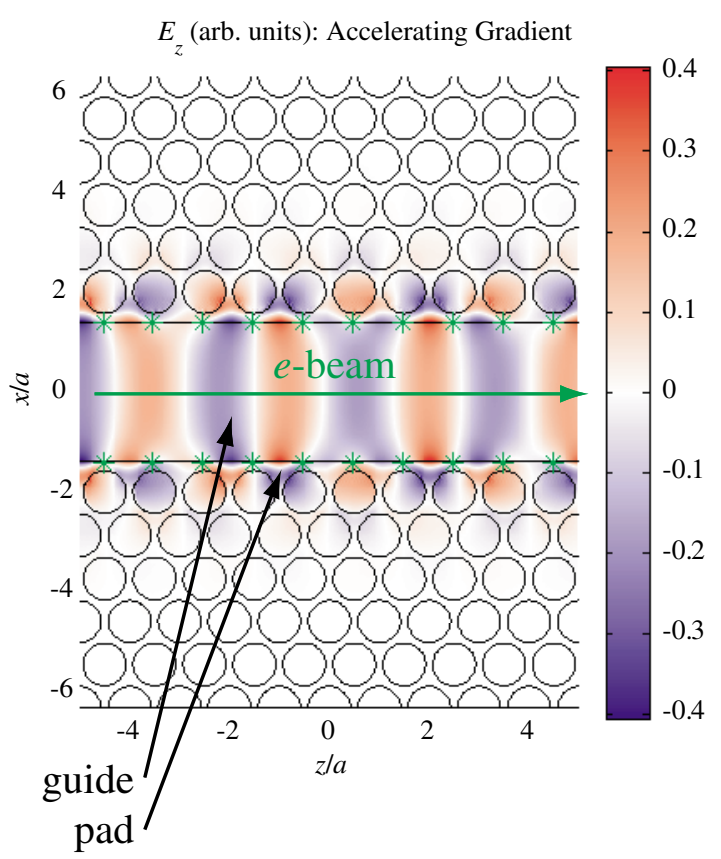

FIG. 5. (Color) An accelerator structure geometry with a speed-of-light waveguide mode. The circles delineate the vacuum holes, and the blue/red coloring denotes the accelerating $E_{z}$ component. Here $w=3.0 a$ and $\delta=0.25 a$, and for this mode $\lambda=2.78 \mathrm{a}$. The asterisks show the damage sites, where the field magnitude in the dielectric is maximized.

By applying the Feynman-Hellmann theorem [11,12], MPB can also compute group velocities for each mode with little additional computational effort.

Using the frequencies and group velocities, we can inductively converge on the longitudinal wave number for which a speed-of-light mode exists. Suppose we have found frequency $\omega^{(n)}$ at wave number $k_{z}^{(n)}$. We also know the slope of the dispersion curve, since the group velocity of this mode is $v_{g}=d \omega /\left.d k_{z}\right|_{k_{z}^{(n)}}$. Approximating the dispersion curve by the linear relationship given by these parameters, we can estimate the frequency and wave number of the speed-of-light mode for the next step using the relations

$$
c k_{z}^{(n+1)}=\omega^{(n+1)}=\omega^{(n)}+v_{g}\left(k_{z}^{(n+1)}-k_{z}^{(n)}\right) .
$$

This yields

$$
k_{z}^{(n+1)}=k_{z}^{(n)}+\frac{\omega^{(n)}-k_{z}^{(n)}}{1-\beta_{g}},
$$

where $\beta_{g}=v_{g} / c$. Since the iterative eigensolver for each step can be seeded with the field configuration from the result of the previous step, successive steps are quite fast, and convergence to a speed-of-light mode is a computationally light task once the initial mode has been found.

For the 2D structure computations, a grid resolution of 32 pixels per lattice constant was used. We used a 
supercell width (transverse to the beam direction) of

$$
W_{s}=[(5 \sqrt{3}+1)+w] a .
$$

Each computation of an accelerating mode required between 6 and $66 \mathrm{~min}$ of CPU time on a $440 \mathrm{MHz}$ Sun Netra t1-105 computer with 256 MB RAM.

\section{ACCELERATING MODE PARAMETERS}

Several parameters characterize the performance of an accelerating mode. The relationship between the input laser power and the accelerating gradient is described by the characteristic impedance [13]. Since our 2D structures only confine modes in one transverse dimension, we normalize the impedance to that of a structure one wavelength high, so

$$
Z_{c}=\frac{E_{\mathrm{acc}}^{2} \lambda}{P_{h}},
$$

where $E_{\text {acc }}$ is the accelerating gradient on axis and $P_{h}$ is the laser power per unit height. We can measure $P_{h}$ from the computation by summing the $z$ component of the Poynting vector over one transverse slice of the mode.

Next, there is the damage factor

$$
f_{D}=\frac{E_{\text {acc }}}{|\mathbf{E}|_{\max }^{\operatorname{mat}}},
$$

where $|\mathbf{E}|_{\max }^{\text {material }}$ is the maximum electric field magnitude anywhere in the dielectric material. Since laser power is ultimately limited by the breakdown threshold $E_{\max }$ of the material, the damage factor is an important measure of the maximum possible accelerating gradient a structure can sustain.

However, $E_{\max }$ is not a constant for a given material; rather, it depends on the laser pulse width. In fact, vacuum laser-driven acceleration schemes seek to take advantage of the favorable scaling of material damage thresholds for ultrafast pulses. Thus these accelerator structures are transmission mode, and a high group velocity $v_{g}$ is desired so that short pulses may be used. The minimum pulse width is the time lag of a laser pulse with respect to a speed-of-light particle bunch as they traverse a structure, so it depends on the structure length. Thus the maximum possible gradient is given by

$$
E_{\mathrm{acc}}^{\max }=f_{D} E_{\max }(\tau)=f_{D} E_{\max }\left(\frac{L}{c}\left[\frac{1}{\beta_{g}}-1\right]\right),
$$

where $\tau$ is the pulse width and $L$ is the length of the structure. We take the damage fluence of the material to vary with the pulse width as $\tau^{1 / 2}$, as is the generally accepted picture for pulses longer than about $10 \mathrm{ps}$ (though deviations from this have been observed for shorter pulses) [1]. The damage threshold $E_{\max }$ thus depends on the pulse width as $\tau^{-1 / 4}$.

We computed accelerating modes in photonic crystal waveguides for a variety of geometries. First, we find the

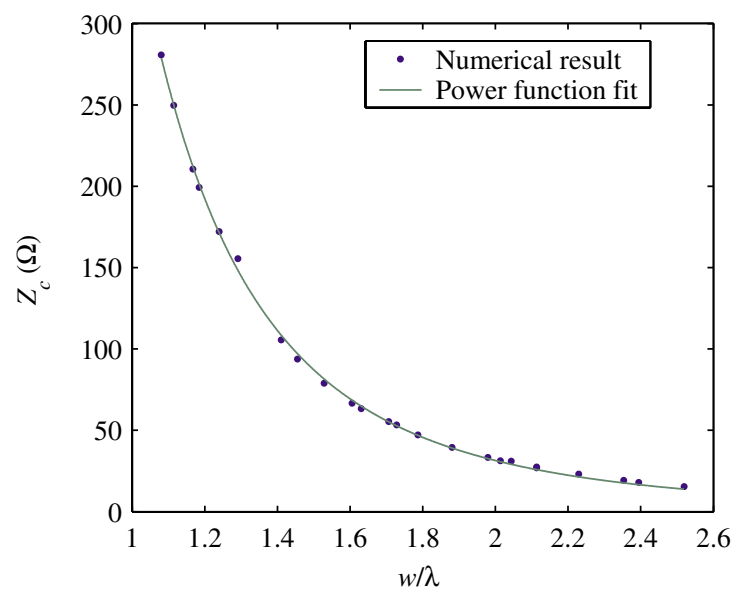

FIG. 6. (Color) Characteristic impedances of 2D photonic crystal waveguide structures. By normalizing the impedance to that of a structure one wavelength high, we obtain a value of $Z_{c}$ in $\Omega$. The data shown here include multiple values of $\delta$ ranging from 0 to $0.25 a$.

characteristic impedance for each geometry and plot $Z_{c}$ versus $w / \lambda$, as shown in Fig. 6.

From a fit to a power law function, we find the dependence $Z_{c} \propto(w / \lambda)^{-3.55}$.

We also find the damage factor and group velocity for each structure geometry. These are plotted in Figs. 7 and 8 , respectively.

The qualitative behavior of these parameters presents a trade-off. As the guide is widened, the damage factor decreases. On the other hand, the group velocity increases, allowing shorter laser pulses to be used, for which the material damage threshold is at a higher field. One of these considerations may dominate the other, or the optimum gradient may be achieved at some intermediate guide width.

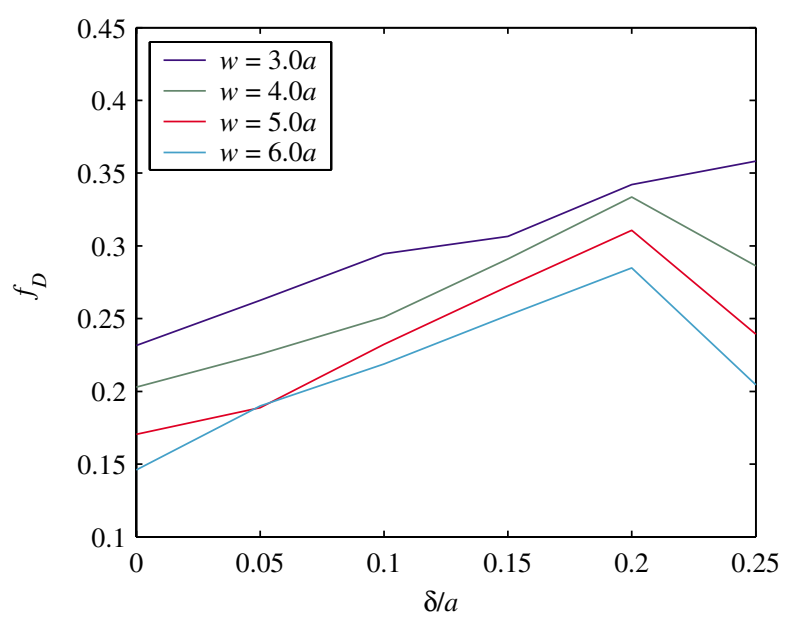

FIG. 7. (Color) Damage factors of various structure geometries. 


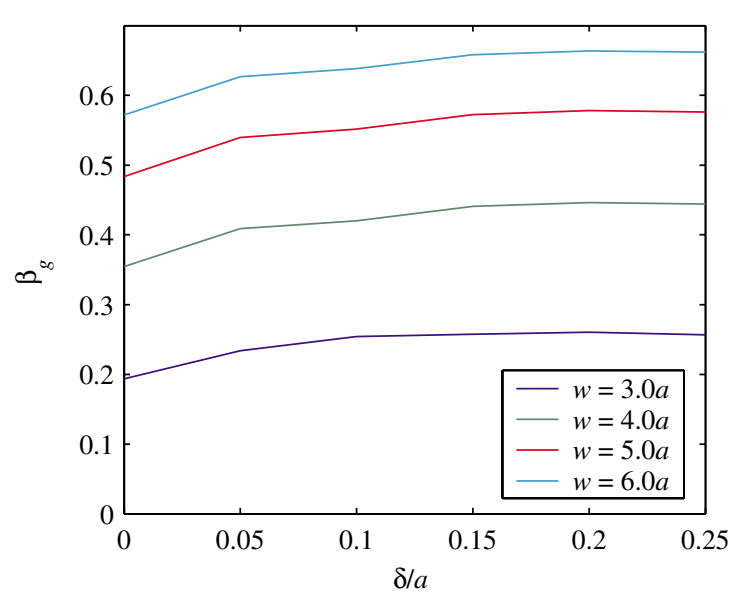

FIG. 8. (Color) Group velocities of accelerating modes for a variety of $w$ and $\delta$ values.

To address this issue, we plot the maximum possible accelerating gradient for each mode using Eq. (1) and the $\tau^{-1 / 4}$ dependence of the damage threshold field, as shown in Fig. 9.

To perform this computation a structure length must be chosen; we make the arbitrary choice of $25 \mathrm{~mm}$. The maximum gradient increases as the structure length is reduced. It appears that the optimum parameters are $w / a=5-6$ and $\delta / a=0.2$.

\section{NUMERICAL EXAMPLE}

We now provide a concrete numerical example of a two-dimensional photonic crystal accelerator structure. As remarked above, for such a structure to be practical we would have to have some method of vertical confinement of the laser mode. In this example let us assume that such

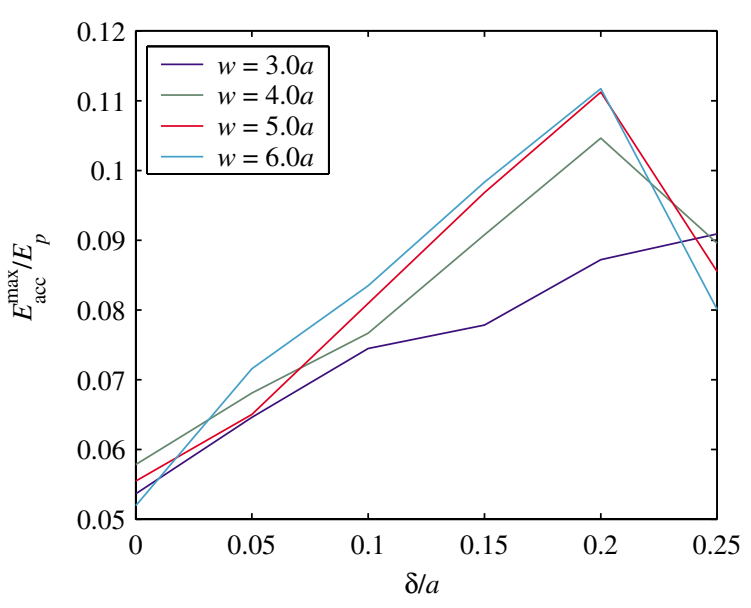

FIG. 9. (Color) The maximum accelerating gradient sustainable by each structure geometry, normalized to the material damage threshold $E_{p}$ for 1 ps pulses. We assume a structure length of $25 \mathrm{~mm}$. a method has been found which confines the mode to one wavelength of height (as assumed in the impedance computations in the previous section) without any distortion of the mode pattern. Although not physical, it will allow us to compute the parameters of the structure.

Referring to the geometric parameters given in Figs. 1 and 3, we let $a=536 \mathrm{~nm}, r=0.427 a=229 \mathrm{~nm}, w=$ $5.0 a=2.68 \mu \mathrm{m}$, and $\delta=0.2 a=107 \mathrm{~nm}$. This structure has a speed-of-light accelerating mode with frequency $\nu=200 \mathrm{THz}$, or wavelength $\lambda=1.50 \mu \mathrm{m}$. The group velocity of this mode is $v_{g}=0.578 c$ and its damage factor is $f_{D}=0.311$. With our assumption on the mode height, the characteristic impedance is $Z_{c}=47.3 \Omega$.

For a structure of length $25 \mathrm{~mm}$, the slippage time of a laser pulse with respect to a speed-of-light electron bunch is 60.8 ps. Assuming a Gaussian time envelope on the laser pulse, we find that a pulse with $55.3 \mathrm{ps}$ full width at half maximum intensity maximizes the average accelerating gradient. The breakdown threshold of silicon for ultrafast near-infrared laser pulses is not known, so we use instead the data from [1] on fused silica at $1053 \mathrm{~nm}$, which gives a breakdown fluence of $10 \mathrm{~J} / \mathrm{cm}^{2}$. This translates to a peak field in the dielectric of $720 \mathrm{MV} / \mathrm{m}$, and from the damage factor stated above we obtain a peak unloaded accelerating gradient of $220 \mathrm{MV} / \mathrm{m}$. The integrated accelerating voltage for the structure is then 4.9 MV, for an average unloaded gradient (reduced from the peak gradient due to the time slippage of the laser pulse) of $190 \mathrm{MV} / \mathrm{m}$.

Finally, if we assume this structure forms a linac with a focusing-defocusing lattice composed of quadrupoles with a field gradient of $1 \mathrm{kT} / \mathrm{m}$ and effective length of $1 \mathrm{~cm}$, then for $5 \sigma$ beam clearance we require an invariant emittance of $2.7 \times 10^{-11} \mathrm{~m}$.

\section{CONCLUSION}

We have found accelerating modes for a set of geometries in a class of $2 \mathrm{D}$ photonic crystal structures. These structures can confine light in an all-dielectric structure and potentially suppress all higher-order guided wakefield modes. We examined parameters of the accelerating modes and analyzed the trade-off between damage factor and group velocity.

While we built a solid intuition for photonic crystal structures, much work remains to be done to fully understand these structures as accelerators. Wakefield losses, the effect of poorer confinement, and coupling structures must be examined. Finally, it is worth pointing out once again that for practical accelerator purposes, the concepts discussed here must be explored in the 3D case.

Fabrication of these structures at optical length scales is likely to pose significant challenges. However, much work on fabricating three-dimensional photonic crystals is underway in the photonics community; one example of a lattice structure is discussed in [14]. The very low 
transverse emittance requirement will pose another experimental challenge. Since collimation of the particle beam will likely be necessary to confine particles to the waveguide, any experimental test of such a structure must have a method of resolving low-charge bunches.

\section{ACKNOWLEDGMENTS}

The author would like to thank R. Siemann and S. Fan for helpful advice. This work has been supported by the Department of Energy Contract No. DE-AC0376SF00515 (SLAC) and by DOE Grant No. DE-FG0397ER41043-II.

[1] B. C. Stuart, M. D. Feit, S. Herman, A. M. Rubenchik, B.W. Shore, and M. D. Perry, J. Opt. Soc. Am. B 13, 459 (1996).

[2] R. L. Byer, T. Plettner, C. Barnes, E. Colby, B. Cowan, R. H. Siemann, and J. E. Spencer, in Proceedings of the 1999 Particle Accelerator Conference, New York (IEEE, Piscataway, NJ, 1999), Vol. 1, pp. 321-324.

[3] T. Plettner, Ph.D. thesis, Stanford University, 2002.
[4] J. D. Joannopoulos, R. D. Meade, and J. N. Winn, Photonic Crystals: Molding the Flow of Light (Princeton University Press, Princeton, NJ, 1995).

[5] X. E. Lin, Phys. Rev. ST Accel. Beams 4, 051301 (2001).

[6] D. R. Smith, S. Shultz, N. Kroll, M. Sigalas, K. M. Ho, and C. M. Soukoulis, Appl. Phys. Lett. 65, 645 (1994).

[7] M. A. Shapiro, W. J. Brown, I. Mastovsky, J. R. Sirigiri, and R. J. Temkin, Phys. Rev. ST Accel. Beams 4, 042001 (2001).

[8] D. F. Edwards, in Handbook of Optical Constants, edited by E. D. Palik (Academic Press, New York, 1985), Vol. 1, p. 547.

[9] R. Keinigs, M. E. Jones, and W. Gai, Part. Accel. 24, 223 (1989).

[10] S. G. Johnson and J. D. Joannopoulos, Opt. Express 8, 173 (2001).

[11] S. G. Johnson, M. Ibanescu, M. A. Skorobogatiy, O. Weisberg, J. D. Joannopoulos, and Y. Fink, Phys. Rev. E 65, 066611 (2002).

[12] D. J. Griffiths, Introduction to Quantum Mechanics (Prentice Hall, Englewood Cliffs, NJ, 1995).

[13] J. R. Pierce, Traveling-wave Tubes (Van Nostrand, New York, 1950).

[14] S. Noda, K. Tomoda, H. Yamamoto, and A. Chutinan, Science 289, 604 (2000). 